\title{
Micro-Kinking of the Long-Period Stacking/Order (LPSO) Phase in a Hot-Extruded $\mathrm{Mg}_{97} \mathrm{Zn}_{1} \mathrm{Y}_{2}$ Alloy
}

\author{
Daisuke Egusa $^{1, *}$, Michiaki Yamasaki $^{2}$, Yoshihito Kawamura ${ }^{2}$ and Eiji Abe ${ }^{1}$ \\ ${ }^{1}$ Department of Materials Science and Engineering, The University of Tokyo, Tokyo 113-8656, Japan \\ ${ }^{2}$ Department of Materials Science, Kumamoto University, Kumamoto 860-8555, Japan
}

\begin{abstract}
Microstructures of a hot-extruded $\mathrm{Mg}_{97} \mathrm{Zn}_{1} \mathrm{Y}_{2}$ alloy containing the long-period stacking/order (LPSO) phase have been investigated by transmission electron microscopy (TEM), particularly focusing on kink-deformed LPSO crystals. It is found that kink-deformation in the LPSO phase grains are mostly characterized by singular straight interfaces across which the host crystals are sharply bended, as being similar to those reported previously. In addition to these common LPSO phase kink-bands, we occasionally find unique microscopic traces at the kinkboundaries, which are composed of multiply segmented kink-interfaces that are sequentially rotated with small angles in a same direction, accomplishing a macroscopic large crystalline bend; i.e., a micro-kinking feature. Occurrence of such micro-kinking may hardly be explained by generation of dipole-pair dislocations in the early stage of kinking, which have been successfully used for phenomenological understanding of kink deformation. [doi:10.2320/matertrans.MI201216]
\end{abstract}

(Received November 26, 2012; Accepted January 24, 2013; Published March 8, 2013)

Keywords: magnesium alloys, long-period stacking/ordered phase, kink deformation, transmission electron microscopy

\section{Introduction}

Recently, Mg alloys with a small addition of $\mathrm{TM}$ (transition-metal atoms) and RE (rare-earth and $\mathrm{Y}$ atoms) have been of focused interests, since they are shown to reveal excellent mechanical properties such as strength greater than $\sim 350 \mathrm{MPa}$ with reasonable ductility, which can be realized by simply applying a conventional hot extrusion process for as-cast ingots. ${ }^{1-3)}$ These excellent mechanical properties of the alloys are believed to be provided by long-period stacking/ordered (LPSO) phase, ${ }^{4-6)}$ through its unique deformation behaviors referred to as "kink deformation" that causes the LPSO crystals to bend sharply by generating definite interfaces (i.e., kink-interfaces). It is generally known that kink deformation may preferably occur for anisotropic crystals owing to their limited slip systems such as an hep crystal. ${ }^{7)}$ Microscopic mechanism of the kink deformation was phenomenologically described by Hess and Barrett ${ }^{8)}$ in terms of a sequential pile up of moving dislocations. In their model, dipole-pair dislocations are generated at buckling flexures, and then the dislocations with a same sign pile up to form a boundary across which the host crystals are sharply bended. Successive generations of such boundary lead to growing of kink bands. Later, Frank and Stroh ${ }^{9)}$ further discussed a mechanism of generating dipole-pair dislocations within grain interiors in the early stage of kinking. The dipole-pair generations and the pile-up behaviors of moving dislocations may fairly explain some representative features relevant to kink deformations; though, microscopic mechanism of kinking has not been fully understood yet because of significant lack of experimental investigations.

Regarding the plastic deformation behaviors of the LPSObased $\mathrm{Mg}-\mathrm{Zn}-\mathrm{Y}$ alloys, Hagihara et al. ${ }^{10,11)}$ found that $(0001)_{\text {hcp }}\langle 1 \overline{2} 10\rangle_{\text {hcp }}$ basal system provides a major slip mode, and other slip systems are restricted due to the anisotropic LPSO structures. ${ }^{12-14)}$ With the stress conditions that the Schmidt factor $\sim 0$ with respect to the relevant slip planes,

*Graduate Student, The University of Tokyo deformation kinks seem to occur alternatively to account for the stress concentration. Kinking is generally known as a minor deformation mode in crystalline materials, ${ }^{15}$ ) but it appears to be an essential mode for the LPSO crystals, for which both the non-basal slip and twinning are strongly suppressed. Typical microstructures of the kink-deformation bands in the LPSO phase grains were shown to be represented by straight boundaries, across which the host crystals are sharply bended with rotational angles from 10 to 90 degrees. ${ }^{10,11,16)}$

During the microstructure investigations of the kinkdeformed LPSO-based Mg-Zn-Y alloy, we have occasionally found unique microscopic traces at the kink-boundaries, which are composed of multiply segmented kink-interfaces that are sequentially rotated with small angles to accomplish a large crystalline bending. This feature is reasonably interpreted as micro-kinking events. In the present work, we will describe details of micro-kinking structures and discuss their characteristics in terms of dislocation dipole-pair generations in the early stage of the kink deformation.

\section{Experimental Procedure}

The nominal composition of the $\mathrm{Mg}-\mathrm{Zn}-\mathrm{Y}$ alloys used in the present work is $\mathrm{Mg}-1$ at $\% \mathrm{Zn}-2$ at\% $\mathrm{Y}$ (hereafter denoted $\mathrm{Mg}_{97} \mathrm{Zn}_{1} \mathrm{Y}_{2}$ ). Master alloy ingot was prepared by high frequency induction melting of pure $\mathrm{Mg}$ (99.99 mass\%), $\mathrm{Zn}$ (99.9 mass\%) and Y (99.9 mass\%) metals in a carbon crucible. Hot-extruded samples were prepared through an extrusion ratio of 10 at $623 \mathrm{~K}$, with a ram speed of $2.5 \mathrm{~mm} \mathrm{~s}^{-1}$ (see Yamasaki et al., ${ }^{17)}$ for further details of sample preparations). Thin foils for transmission electron microscopy (TEM) observations were prepared by standard argon ion milling. Microstructures of the alloys were investigated with a JEM-2010 HC operating at $200 \mathrm{kV}$.

\section{Results}

Figure 1(a) shows a bright-field (BF) TEM image obtained at the kink-band in the hot-extruded $\mathrm{Mg}_{97} \mathrm{Zn}_{1} \mathrm{Y}_{2}$ alloy, with 

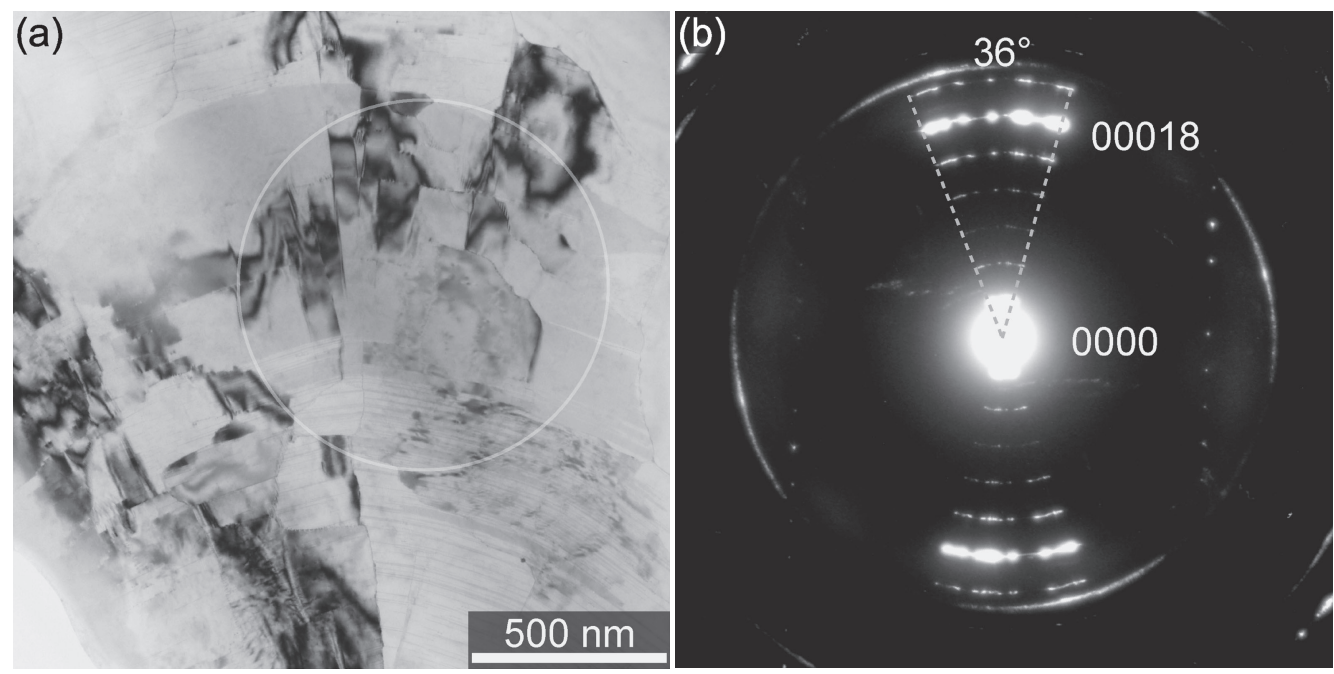

Fig. 1 (a): Bright-field TEM image obtained from a hot-extruded $\mathrm{Mg}_{97} \mathrm{Zn}_{1} \mathrm{Y}_{2}$ alloy. (b): Electron diffraction pattern obtained from the white-circle area in (a).
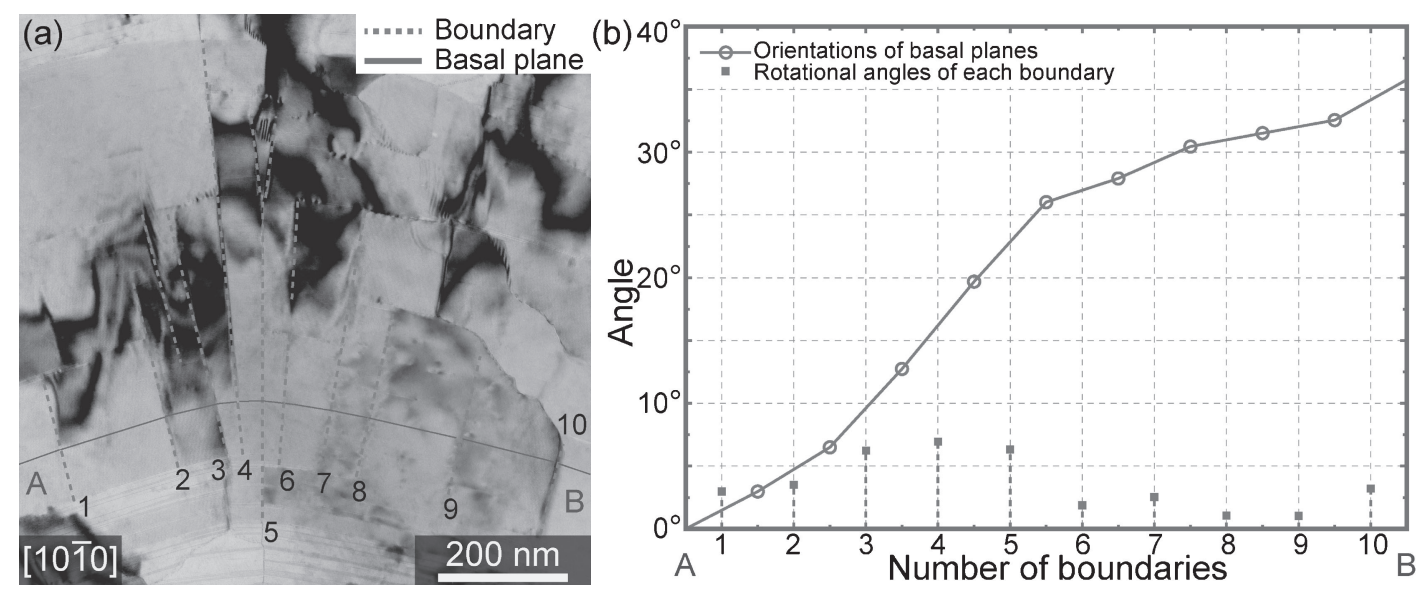

Fig. 2 (a): Bright-field TEM image of the kink-band in a LPSO phase grain. Continuous trace of basal planes across the domains is shown by a solid line, and dotted lines represent the kink interfaces, as numbered from 1 to 10. (b): Plot of rotational angles of each kink interface.

the incident beam nearly along $[10 \overline{1} 0]$ axis of the hep-Mg matrix. In the image, a large number of kink interfaces are formed, dividing the LPSO phase grain into small distinct domains which are sequentially rotated in a same direction from left to right. This is confirmed by the corresponding electron diffraction pattern in Fig. 1(b), where the $000 l$ reflections of the 18R-type LPSO crystals appear by rotating with respect to the common $[10 \overline{1} 0]_{\text {hep }}$ axis up to about 36 degrees. Therefore, these micro-domain features are reasonably interpreted as micro-kinking, which is essentially similar to those referred to as "sub-kink bands". ${ }^{16)}$

Figure 2(a) shows a magnified BF image of the microkinking region in the LPSO phase grain, in which the basal planes and the kink interfaces of each domain are represented by solid lines and dotted lines, respectively. It is seen that the distances between the kink interfaces are about several tens of nanometers apart from each other, and most of the interfaces are segmented and terminated within the LPSO crystals. By tracing the basal planes across the domains, the sequential rotation angles can be successfully measured, and the results are plotted in Fig. 2(b). It is clearly confirmed that all the basal planes rotate only in a clockwise along A to B in Fig. 2(a), with the relevant rotational angles ranging from 1-7 degrees. These features may apparently be similar to the microtwinning bands in some Mg alloys. ${ }^{18,19)}$ However, it should be noted in particular that the various rotation angles observed in Fig. 2(b) can never be explained by the primary and secondary twining of the LPSO crystals, suggesting that the micro-kinking is definitely different from the twinning event.

Figures 3(a) and 3(b) show the BF images obtained from a part of Fig. 2(a), taken with two-beam conditions of $g=1 \overline{2} 10$ and $\mathrm{g}=00018$; i.e., $a$-excitation and $c$-excitation of the $\alpha$-Mg structure, respectively. Note that these excitation conditions just confirmed whether or not each of the dislocations possesses the relevant components. Namely, the precise burgers vectors of the dislocations can hardly be determined only from the present $g \cdot b$ analysis (e.g., the burgers vectors can either be $\langle 1 \overline{2} 10\rangle$ or $\langle 1 \overline{1} 00\rangle$ when successfully excited with $\mathrm{g}=1 \overline{2} 10$ ). Because of this situation, we hereafter denote these dislocations as $a^{\prime}$-dislocations and $c^{\prime}$-dislocations, which do not represent any specific burgers vectors. In the image of Fig. 3(a), it is found that a number of $a^{\prime}$-dislocations are imaged as dark spots, which are semi-regularly arrayed along the kink interface. ${ }^{16)}$ Localized appearances of the contrast 


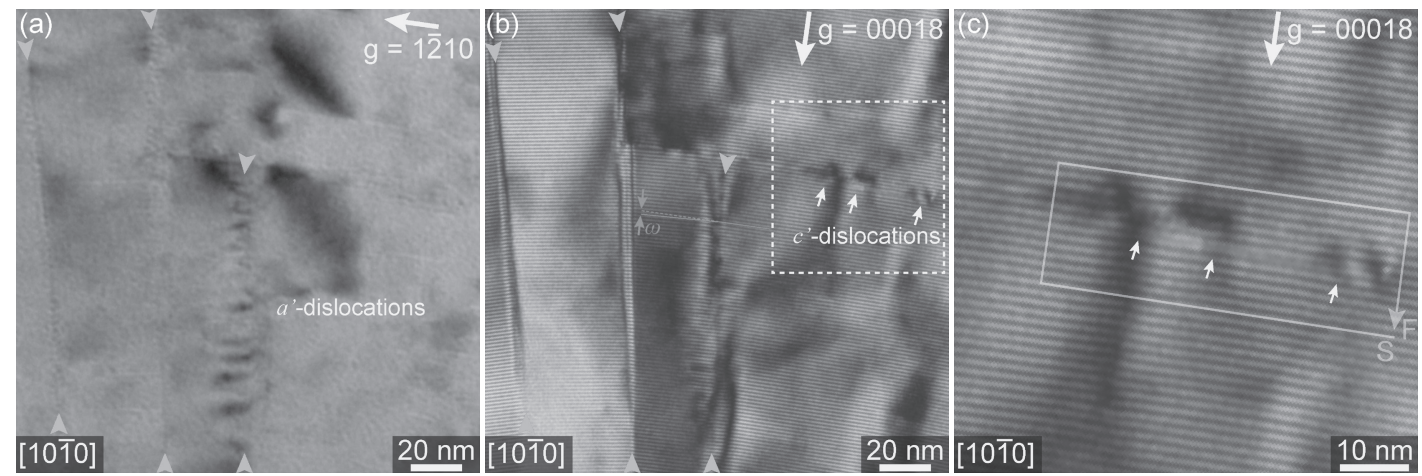

Fig. 3 (a), (b): Bright-field TEM images of the kink interfaces with two-beam conditions; (a) $g=1 \overline{2} 10$ and (b) $g=00018$, respectively. Gray arrowheads indicate the positions of the kink interfaces, and white arrowheads in (b) represent $c^{\prime}$-dislocations. (c): Enlarged view of white-framed area in (b). Circuit with white line indicates the occurrences of extra half planes along stacking direction.

indicate that the dislocations are viewed as nearly edge-on conditions. It is noteworthy here that the $a^{\prime}$-dislocations are not effectively excited at the adjacent kink interfaces in Fig. 3(a); this is perhaps due to the slight misorientations between the micro-kink domains. In fact, we indeed confirmed that the similar array of $a^{\prime}$-dislocations was also emerged for other kink interfaces by slightly tilting the specimen to alter the excitation conditions. The relationship between dislocation densities at the interface and rotational angles across the kink interface is expressed by the equation;

$$
\tan \omega=L /|\boldsymbol{b}|
$$

where the $\omega$ is a rotational angle, $L$ is an average interval length of dislocations, and $|\boldsymbol{b}|$ is an absolute value of burgers vector, respectively. For the kink interface in Fig. 3(b), $\omega$ indicated by gray lines and $L$ are measured to be about 2 degrees and $9 \mathrm{~nm}$, respectively. Assuming $\boldsymbol{b}=1 / 3\langle 1 \overline{2} 10\rangle$, $|\boldsymbol{b}|$ becomes $0.32 \mathrm{~nm}$ and hence the relevant $\omega$ is calculated to be 1.9 degrees, which is almost equivalent to the observed value of 2 degrees.

Characteristic arrays of $c^{\prime}$-dislocations are also formed in the vicinity of kink interfaces composed of the $a^{\prime}$ dislocations, as indicated by white arrows in Fig. 3(b). Similar $c^{\prime}$-dislocation arrays are frequently observed in the present kink-deformed specimen, even though $c^{\prime}$-dislocations have been rarely observed for $\mathrm{Mg}$ alloys reported previously. ${ }^{20)}$ In Fig. 3(b), note that the $c^{\prime}$-dislocations aligned parallel to the basal planes, whose extensions will converge around the termination point of the kink-interface segment embedded within the grain interiors. It is also noteworthy that the $c^{\prime}$-dislocation arrays can be traced by the relevant extra half planes (Fig. 3(c)), producing distinct line contrast along the basal planes, as frequently seen in Fig. 2(a). On the basis of this feature, we consider that these $c^{\prime}$-dislocations play an important role for the toughness of the LPSO-Mg alloys, as discussed below. Generally, it may naturally be expected that microcrack generations are an unavoidable consequence after the kink deformation, as schematically shown in Fig. 4(a). ${ }^{21)}$ In fact, such microcracks have been frequently observed for several kink-deformed materials, e.g., hexagonal-BN, ${ }^{22}$ $\mathrm{SiC}^{23)}$ and $\mathrm{Ti}_{3} \mathrm{SiC}_{2} .{ }^{24)}$ For the LPSO crystals, however, occurrence of microcracks seem to be effectively suppressed by generating the arrays of $c^{\prime}$-dislocation, as illustrated in Fig. 4(b). Although a mechanism for emitting these
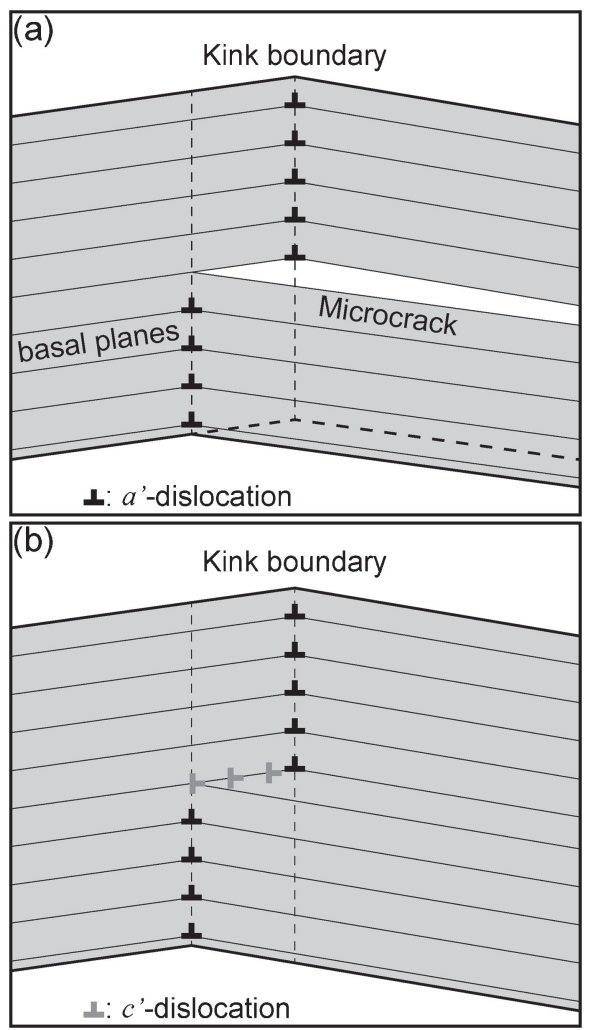

Fig. 4 Schematic illustrations of microcrack formation in a kink band. (a): Discontinuous rotation at the terminated interface causes a microcrack. (b): Array of $c^{\prime}$-dislocations between the terminated interfaces compensates the discontinuous rotation of the crystal, suppressing occurrence of the microcrack.

dislocations (perhaps within grain interiors) is not fully elucidated, the dislocation distributions in Fig. 4(b) fairly well explain the observed features as exemplified in Fig. 3. In any cases, such unique dislocation arrangements would contribute for avoiding microcracking and hence realizing a reasonable toughness of the LPSO-Mg alloys.

We also find a micro-kinking feature in a largely bended kink band. Figure 5(a) shows a BF image of a kink-deformed LPSO phase grain bended from bottom to top, whose rotational angle is estimated to be about 92 degrees from the electron diffraction patterns at $\mathrm{X}$ and $\mathrm{Y}$ regions. Looking at details of micro-kinks in Fig. 5(b), it is found that the microscopic features are almost similar to those described in 

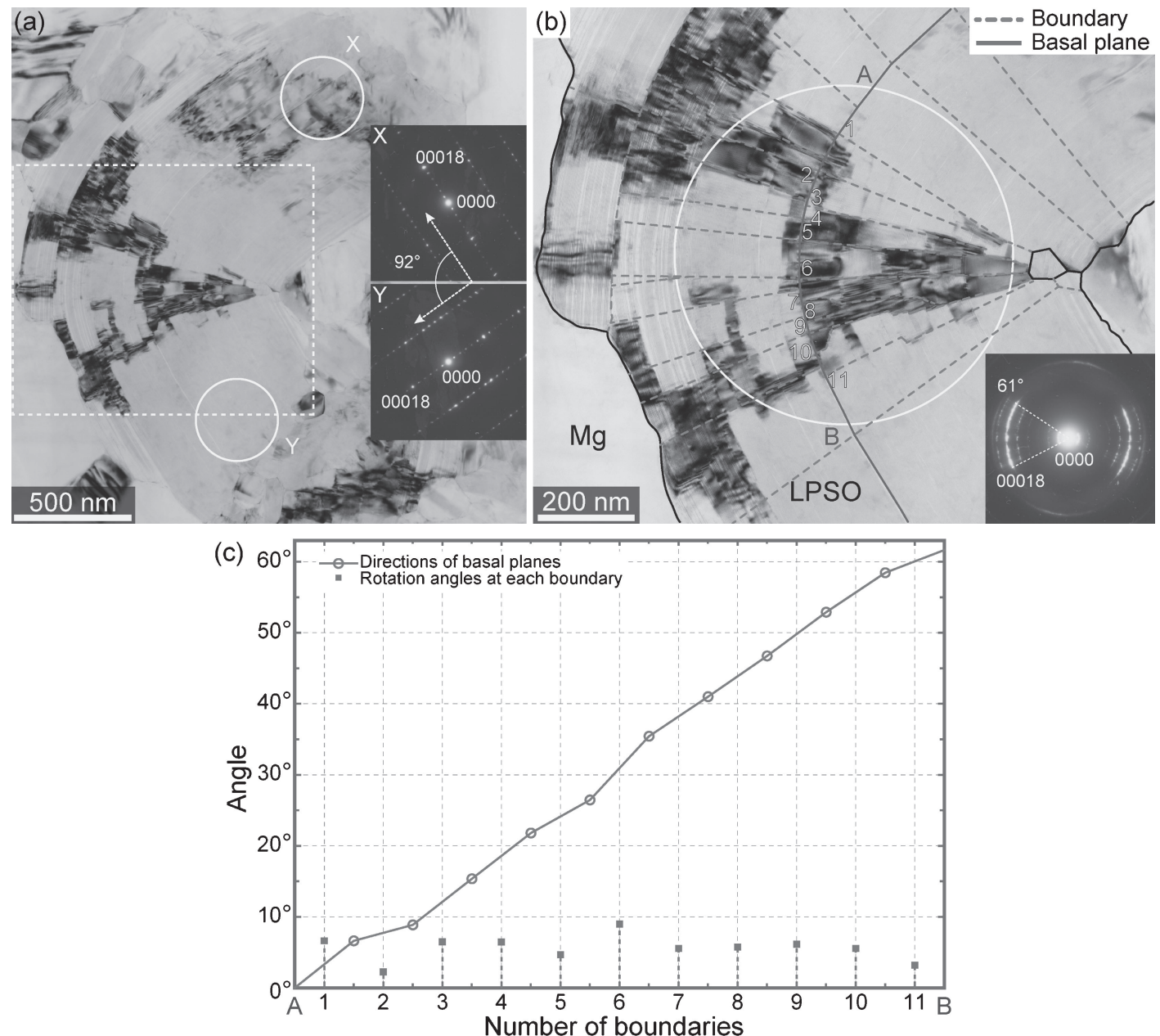

Fig. 5 (a) Bright-field TEM image of the kink band taken nearly along [1210] direction. Electron diffraction patterns were obtained from the region represented as $\mathrm{X}$ and $\mathrm{Y}$, respectively. (b) Magnified image of the rectangle region in (a). Interfaces and basal planes are represented by gray dotted lines and a solid line, respectively. Electron diffraction pattern was obtained from the white-circled area. (c): Plot of rotational angles of each kink interface.

Fig. 2; domains divided by kink interfaces are sequentially rotated with small angles and all in a same direction, as confirmed by the relevant plot shown in Fig. 5(c). Accordingly, it is shown that micro-kinking is able to achieve a wide range of total bend angles, as observed for common kinking traced by singular sharp interfaces.

\section{Discussion}

We discuss the characteristics of the present micro-kinking in the LPSO crystals in terms of existing kink-deformation models. ${ }^{7-9)}$ In the early stage of kink-deformation, one of the prominent role of dislocations are believed to be dipole-pair generations within a grain interiors, as illustrated in Fig. 6. As a consequence, a pair of kink interfaces composed of opposite edge dislocations will be generated, revealing a stepwise macroscopic feature of the kink deformation bands, as shown in Fig. 6. Note that, when all the kink interfaces are generated from dipole-pair dislocation walls, total rotation (bend) angles of the stepwise kink bands should appear to be zero. For the present micro-kinking structure observed in the largely bended kink bands, no set of dipole-pair dislocation walls was identified, and all the micro-kink domains are rotated along the same direction. Therefore, the micro-

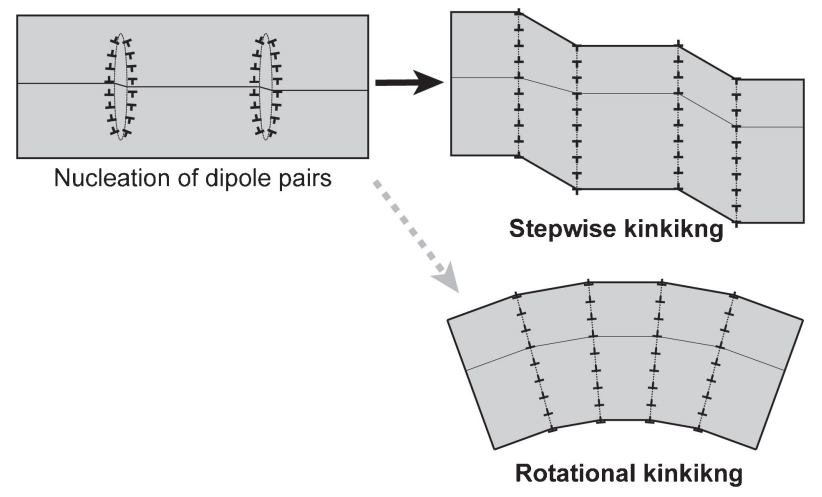

Fig. 6 Schematic illustrations of kink deformation: (left) Nucleation of dislocation dipole pairs occurs within a grain interior. (right-top) Dislocation walls moved apart and form sharp interfaces with a stepwise shape. (right-bottom) Array of kink interfaces observed in the present study. Rotational kinking results in the relevant interfaces with a same rotational direction.

kinking is hardly expected from the dipole-pair initiation models. Similar discussion was also made for micro-kinking feature in a kink-deformed $\mathrm{Ti}_{3} \mathrm{SiC}_{2}{ }^{24)}$ Although there is no idea on how the dislocations have been generated in the early stage of micro-kinking in the LPSO crystals, it is worthwhile 
to note that the micro-kink regions composed of a high density dislocation walls (i.e., kink interface segments) may be favored for recovering and/or recrystallization events during hot-extrusion process at sufficiently high temperatures (623 K).

So far, kink deformations have been confirmed for various deformation conditions; i.e., single- or poly-crystalline specimens, compression or hot-extrusion, etc. It is expected that, in a strict sense, applied local stress in each of the grains could be considerably different depending on the conditions. On this regard, interesting kink deformation behaviors were reported for mineral crystals (muscovite aggregates), ${ }^{25)}$ by performing compression and torsion mechanical tests. When the sample is deformed in torsion, a unique kink-within-kink feature appears, which has never been realized in the compressed samples. This result clearly demonstrates that local stress fields significantly affect the microscopic features of kink bands. It may be presumed that, for the LPSO crystals, dipole-pair driven stepwise kinking preferentially occurs when the grains are attributed to a simple uniaxial compression stress. ${ }^{10,11)}$ While, complicated kink deformations including micro-kinking/rotational-kinking may occur when the grains are under multiple stress conditions such as represented by shear, bending, torsion, etc. Further investigations are essentially necessary to clarify the microscopic mechanism of kink deformations of crystalline materials.

\section{Conclusions}

Microstructures of the LPSO phase kink bands formed in $\mathrm{Mg}_{97} \mathrm{Zn}_{1} \mathrm{Y}_{2}$ alloys have been investigated based on TEM observations. We have found the unique microscopic features at the kink-boundaries, which are composed of multiply segmented kink-interfaces with small rotational angles; i.e., a micro-kinking features. The main results are summarized as follows.

(1) Electron diffraction patterns and BF-TEM images showed the occurrences of multiple kink interfaces which divide the LPSO phase grain into small domains. Based on the traces of the basal planes across the domains, it is confirmed that all the basal planes of the each domain rotate in a same direction with the relevant rotational angles smaller than 10 degrees, accomplishing a macroscopic large crystalline bend. These microkinking features are apparently similar to the microtwinning bands in Mg alloys; however, the presence of the various rotational angles suggests that the microkinking is different from the twinning phenomena.

(2) Characteristic arrays of the dislocations were found at the kink interfaces. The $a^{\prime}$-dislocations are semiregularly arrayed along the kink-interfaces with the various densities so as to rotate LPSO crystals across the kink-interfaces. The $c^{\prime}$-dislocations are aligned parallel to the basal planes, whose extensions will converge around the termination point of the kinkinterface segment embedded within the grain interiors. The characteristic arrangements of the $c^{\prime}$-dislocations are expected to suppress the occurrence of microcracking, realizing the reasonable toughness of the LPSO-Mg alloys.
(3) Regarding crystalline rotations during kink deformation, the dipole-pair initiation model hardly explains the present micro-kinking features which do not show any traces of dipole-pair dislocation walls. Although we have no idea on details of micro-kinking mechanism, the present micro-kinking might have occurred when the grains are under multiple stress conditions such as represented by shear, bending, torsion, etc.

\section{Acknowledgement}

This work was supported by a Grant-in-Aid for Scientific Research on Priority Areas "Synchro-LPSO Structures" from the Ministry of Education, Culture, Sports, Science and Technology (MEXT), Japan and the Kumamoto Prefecture CREATE Project from the JST. A part of this work was conducted in Research Hub for Advanced Nano Characterization, The University of Tokyo, supported by MEXT. D. E was supported as a Japan Society for the Promotion of Science (JSPS) research fellows.

\section{REFERENCES}

1) Y. Kawamura and M. Yamasaki: Mater. Trans. 48 (2007) 2986-2992.

2) S. Yoshimoto, M. Yamasaki and Y. Kawamura: Mater. Trans. 47 (2006) 959-965.

3) T. Itoi, T. Seimiya, Y. Kawamura and M. Hirohashi: Scr. Mater. 51 (2004) 107-111

4) Y. Kawamura, K. Hayashi, A. Inoue and T. Matsumoto: Mater. Trans. 42 (2001) 1172-1176.

5) A. Inoue, Y. Kawamura, M. Matsushita, K. Hayashi and J. Koike: J. Mater. Res. 16 (2001) 1894-1900.

6) E. Abe, Y. Kawamura, K. Hayashi and A. Inoue: Acta Mater. 50 (2002) 3845-3857.

7) E. Orowan: Nature (London) 149 (1942) 643-644.

8) J. B. Hess and C. S. Barrett: Trans. Metall. Soc. AIME 185 (1949) 599606.

9) F. C. Frank and A. N. Stroh: Proc. Phys. Soc. London Sect. B 65 (1952) 811-821.

10) K. Hagihara, N. Yokotani and Y. Umakoshi: Intermetallics 18 (2010) 267-276.

11) K. Hagihara, Y. Sugino, Y. Fukusumi, Y. Umakoshi and T. Nakano: Mater. Trans. 52 (2011) 1096-1103.

12) E. Abe, A. Ono, T. Itoi, M. Yamasaki and Y. Kawamura: Philos. Mag. Lett. 91 (2011) 690-696.

13) H. Yokobayashi, K. Kishida, H. Inui, M. Yamasaki and Y. Kawamura: Acta Mater. 59 (2011) 7287-7299.

14) D. Egusa and E. Abe: Acta Mater. 60 (2012) 166-178.

15) A. G. Zhou and M. W. Barsoum: Metall. Mater. Trans. A 40 (2009) 1741-1756.

16) X. H. Shao, Z. Q. Yang and X. L. Ma: Acta Mater. 58 (2010) 47604771.

17) M. Yamasaki, K. Hashimoto, K. Hagihara and Y. Kawamura: Acta Mater. 59 (2011) 3646-3658.

18) H. Yoshinaga, T. Obara and S. Morozumi: Mater. Sci. Eng. 12 (1973) 255-264.

19) Q. Yu, J. Zhang and Y. Jiang: Philos. Mag. Lett. 91 (2011) 757-765.

20) J. Koike, T. Kobayashi, T. Mukai, H. Watanabe, M. Suzuki, K. Maruyama and K. Higashi: Acta Mater. 51 (2003) 2055-2065.

21) A. N. Stroh: Philos. Mag. 3 (1958) 625-646.

22) S. Turan and K. M. Knowles: Phys. Status Solidi A 150 (1995) 227237.

23) H. Suematsu, T. Suzuki, T. Iseki and T. Mori: J. Am. Ceram. Soc. 74 (1991) 173-178.

24) M. Barsoum, L. Farber and T. El-Raghy: Metall. Mater. Trans. A 30 (1999) 1727-1738

25) S. Misra and J.-P. Burg: Tectonophysics 548-549 (2012) 22-33. 